\title{
Caracterização de resíduos de rochas ornamentais provenientes de diferentes processos de corte e beneficiamento
}

\author{
Characterization of ornamental rock wastes \\ from different cutting and beneficiation \\ processes
}

Max M. Aguiar Santos; A.Z. Destefani; J.N.F. Holanda

Grupo de Materiais Cerâmicos - GMCer - LAMAV/CCT/UENF - 28013-602, Campos dos Goytacazes, RJ. e-mail: max-masters@ hotmail.com; andestefani@hotmail.com; holanda@uenf.br

\section{RESUMO}

O Brasil está entre os seis maiores responsáveis pela produção mundial de rochas ornamentais, sendo o centro produtor mais rico em granito. $\mathrm{O}$ setor de rochas ornamentais gera durante os processos de corte e beneficiamento enormes quantidades de resíduos sólidos poluentes, de tal modo que as quantidades de tais resíduos são esperadas para aumentar cada vez mais. O estado do Espírito Santo é o maior produtor e exportador nacional de rochas ornamentais e, nos processos de corte e beneficiamento são geradas grandes quantidades de resíduos sólidos que precisam ser reaproveitados, do contrário podem causar danos de difícil remediação ao meio ambiente. Dependendo do tipo de rocha e do tipo de processo de corte e beneficiamento, resíduos com distintas características podem ser gerados. Neste contexto, o presente trabalho objetiva caracterizar quatro resíduos de rochas ornamentais gerados em distintos processos de corte e beneficiamento denominados de resíduos granalha, dimantado, levigação e polimento. As amostras dos resíduos de rochas ornamentais foram caracterizadas em termos de composição química, morfologia, distribuição de tamanho das partículas, massa específica real e análise termogravimétrica (ATG/DTG). Os resultados experimentais indicaram que os resíduos estudados apresentam diferentes características químicas e físicas. Os resíduos levigado, polimento e granalha são ricos em óxidos fundentes $\left(\mathrm{K}_{2} \mathrm{O}, \mathrm{CaO}\right.$ e $\left.\mathrm{Fe}_{2} \mathrm{O}_{3}\right)$, enquanto que o resíduo diamantado é rico em $\mathrm{SiO}_{2}$. Os resíduos também apresentam distintos comportamentos granulométrico e térmico.

Palavras-chave: Resíduo de rocha ornamental, caracterização, reciclagem.

\begin{abstract}
Brazil is among the top six worldwide responsible by the production of ornamental rocks, being the richest production center in granite. The sector of ornamental rocks generates during the cutting and beneficiation processes huge amounts of pollutants solid waste, in such way that the amounts of such solid wastes are expected to increase more and more. The Espírito Santo state is the largest national producer and exporter of ornamental rocks. In cutting and beneficiation processes of ornamental rocks huge amounts of solid wastes that need to be reused are generated, otherwise they cause damage of difficult remediation to the environment. Depending on the type of rock and the type of cutting and beneficiation process, wastes with different characteristics can be generated. In this context, this work aims to characterize four ornamental rocks wastes generated in different cutting and beneficiation processes denominated as shot waste, diamond waste, levigation waste, and polishing waste. The ornamental rock wastes samples were characterized in terms of chemical composition, morphology, particle size distribution, real specific mass, and thermal analysis (TGA/TGD). The experimental results indicated that the studied ornamental rock wastes presented different chemical and physical characteristics. The levigation, polishing, and shot wastes are rich in $\mathrm{K}_{2} \mathrm{O}, \mathrm{CaO}$ and $\mathrm{Fe}_{2} \mathrm{O}_{3}$, while the diamond waste is rich in $\mathrm{SiO}_{2}$. The ornamental rock wastes also presented different granulometric and thermal behaviors.
\end{abstract}

Keywords: Ornamental rock waste, characterization, recycling. 


\section{INTRODUÇÃO}

As rochas são classificadas cientificamente de acordo com suas composições químicas e mineralógicas e, também, com base em sua textura. As rochas ornamentais e de revestimentos, também chamadas de rochas dimensionadas (dimension stones), abrangem tipos litológicos que podem ser extraídos em forma de blocos e transformados em placas cortadas e beneficiadas [1-2]

Os granitos são rochas plutônicas ou intrusivas granulares formadas, essencialmente, por quartzo e feldspato, podendo conter também minerais acessórios, como biotita e moscovita. Eles são resultantes do lento processo de resfriamento e solidificação do magma [3]]. Já os mármores são rochas metamórficas contendo mais de $50 \%$ de carbonatos (calcita ou dolomita) formados pela recristalização de uma rocha carbonatada [ $\underline{3}-\underline{4}]$.

Em 2011 o Brasil foi classificado como o sexto maior produtor mundial de rochas ornamentais, o sétimo maior exportador em volume físico total e o terceiro maior exportador de blocos de granitos e produtos de ardósia [5]. O Estado do Espírito Santo é o principal produtor e exportador nacional de rochas ornamentais, principalmente de rochas graníticas, cuja produção em 2011 foi da ordem de 3,6 milhões de toneladas []]. A cadeia produtiva da indústria de rochas ornamentais tem uma grande importância econômica e social no país. No entanto, uma quantidade vultosa de resíduos é gerada tornando seu destino final um problema ambiental, pois contaminam diretamente os rios e o próprio solo, além da desfiguração da paisagem, o que vem preocupando as autoridades e a população.

O sistema de desdobramento de blocos de rochas ornamentais para a produção de chapas gera uma quantidade significativa de rejeitos na forma de lama, de 20 a $25 \%$ da massa dos blocos, geralmente constituída de água, granalha, cal e rocha moída, que após o processo, são lançadas no meio ambiente em forma de rejeito, ocupando espaços ao ar livre [7].

O beneficiamento das rochas ornamentais é constituído por três tipos distintos de processos [7]]: serragem em teares, serragem em talha-blocos e acabamento de superfície. O processo de serragem nos teares é auxiliado por uma polpa de água, cal e granalha, despejada continuamente sobre a carga, para otimização do corte e resfriamento das lâminas. Talha-blocos são equipamentos de serragem com discos diamantados, capacitados para cortes de grande profundidade, cuja maior utilização é voltada para produtos padronizados (lajotas). Os equipamentos com discos diamantados, capacitados para cortes mais rasos são chamados talhachapas e muito utilizados nas marmorarias. Após a serragem, o passo seguinte do beneficiamento é o acabamento final das chapas e outras peças, através de levigamento, polimento e lustro ou apicoamento e flameamento. O levigamento ou desbaste representa o desengrossamento das chapas, com a criação de superfícies planares e paralelas. O polimento produz o desbaste fino da chapa e o fechamento dos grãos minerais, criando uma superfície lisa, opaca e mais impermeável que a de uma face natural da mesma rocha. O lustro é aplicado no sentido de se imprimir brilho à superfície da chapa, produzido pelo espelhamento das faces dos cristais constituintes da rocha [7]. Os resultados do polimento e lustro são definidos pelo brilho, fechamento e espelhamento das chapas, podendo-se aferir o brilho através da acuidade visual ou com uso de aparelhos (glossmeter) [8].

Aliado aos problemas ambientais causados pela deposição dos resíduos no meio ambiente, algumas características específicas destes resíduos sólidos como granulometria fina e composição química prédefinida vislumbram potencialidades para sua utilização como material na construção civil. De fato, existe extensa literatura [9-13] relacionada à caracterização e uso de resíduos sólidos provenientes da indústria de rochas ornamentais em materiais para a área. No entanto, estes trabalhos, via de regra, utilizam o resíduo de rocha ornamental de um único tanque de decantação, ou seja, sem a separação dos resíduos das diferentes etapas do processo de beneficiamento.

O presente trabalho tem como objetivo caracterizar quatro resíduos gerados na indústria de rochas ornamentais, provenientes de diferentes processos de corte e beneficiamento de blocos. Ênfase especial é dada sobre a caracterização química, física e térmica dos diferentes resíduos de rochas ornamentais.

\section{MATERIAIS E MÉTODOS}

Neste trabalho foram utilizados quatro resíduos sólidos de rochas ornamentais gerados em diferentes processos de corte e beneficiamento de rochas. Os resíduos utilizados são: I) resíduo de rocha ornamental proveniente do corte com lâmina metálica, utilizando granalha de aço, denominado de resíduo granalha; II) resíduo de rocha ornamental proveniente do corte com fio diamantado, denominado de resíduo diamantado; III) resíduo de rocha ornamental proveniente da etapa de levigação (desengrossamento das chapas), denominado de resíduo levigação; e IV) resíduo de rocha ornamental proveniente da etapa de polimento desbaste fino da 
chapa), denominado de resíduo polimento. Deve-se ressaltar que os resíduos utilizados foram coletados separadamente nos diferentes processos de corte e beneficiamento (serragem, levigação e polimento) de rochas ornamentais.

Os resíduos são provenientes da região sul do estado do Espírito Santo, mais especificamente do município de Cachoeiro de Itapemirim. Os resíduos foram coletados obedecendo aos procedimentos de amostragem de resíduos sólidos da NBR 10007 [14]. Os resíduos coletados foram submetidos à secagem em estufa a $105^{\circ} \mathrm{C}$ por um período de $24 \mathrm{~h}$, destorroado e homogeneizado para ser utilizado.

As amostras de resíduos de rochas ornamentais foram caracterizadas sob diversos aspectos: composição química, análise granulométrica, massa específica real e análise termogravimétrica (ATG/DTG).

A composição química dos resíduos, em forma de óxidos, foi obtida por meio da técnica de análise química por espectroscopia de raios-X de energia dispersiva (EDX), em um equipamento marca Shimadzu, modelo EDX-700.

Os ensaios de granulometria dos resíduos de rochas ornamentais foram realizados através de uma combinação de peneiramento e sedimentação, de acordo com os procedimentos da NBR 7181[15].

A determinação da massa específica real das partículas dos resíduos foi determinada de acordo com os procedimentos da norma técnicas NBR NM 52 [16].

A morfologia das partículas dos resíduos foi observada via microscopia eletrônica de varredura usando um microscópio eletrônico de varredura marca Shimadzu, modelo SEM SSX-550, operando em 15 kV, após cobertura das partículas com uma fina camada de ouro.

Os ensaios de análise termogravimétrica (ATG/DTG) foram realizados em um analisador térmico simultâneo marca BP Engenharia, modelo RB 3000, com taxa de aquecimento de $5^{\circ} \mathrm{C} / \mathrm{min}$.

\section{RESULTADOS}

A Tabela 1 apresenta as composições químicas dos resíduos de rochas ornamentais estudados. Verifica-se que todos os resíduos são quimicamente materiais sílico-aluminosos, devido aos elevados teores de sílica e alumina na faixa de $72,921-95,316 \%$.

Os resultados da Tabela 1 também indicam importantes diferenças nas composições químicas dos resíduos. Nota-se que o resíduo diamantado apresenta o mais alto teor de sílica da ordem de 92,058 \%, mas isento de óxido de ferro. A origem da sílica pode estar relacionada a diversas origens como, por exemplo, aos feldspatos, minerais micáceos (biotita e mica muscovita) e sílica livre presente em rochas silicáticas [3]. Por outro lado, os resíduos levigação, polimento e granalha são ricos em óxidos fundentes $\left(\mathrm{K}_{2} \mathrm{O}+\mathrm{Fe}_{2} \mathrm{O}_{3}+\mathrm{CaO}\right)$, enquanto que o resíduo diamantado apresenta baixo teor de fundentes. É importante destacar que o óxido de cálcio $(\mathrm{CaO})$ e óxido de ferro $\left(\mathrm{Fe}_{2} \mathrm{O}_{3}\right)$ nos resíduos levigação, polimento e granalha são oriundos da cal utilizada como lubrificante e granalha como abrasivo no processo de beneficiamento. A presença de óxidos fundentes nos resíduos é importante, pois tornam esses resíduos atrativos para uso como matéria-prima alternativa na formulação de massas cerâmicas para fabricação de materiais como pisos vitrificados e porcelanas diversas [12, 17-19]. Neste caso, o resíduo de rocha ornamental poderia substituir o material fundente natural normalmente utilizado, no caso feldspatos sódico e potássico. Deve-se ressaltar ainda, que os resíduos estudados ricos em $\mathrm{SiO}_{2}+\mathrm{Al}_{2} \mathrm{O}_{3}+\mathrm{Fe}_{2} \mathrm{O}_{3}(80,980-95,316 \%)$ apresentam grande potencial para serem utilizados na confecção de argamassas para a construção civil [20,21].

A Figura 1 apresenta as curvas de distribuição de tamanho das partículas dos resíduos de rochas ornamentais estudados, cujos dados granulométricos estão resumidos na Tabela 2. Pode-se observar que todos os resíduos apresentam larga faixa de tamanho das partículas: 7,9 - 9,8\% na fração argila $(<2 \mu \mathrm{m}), 18,4-$ $81,8 \%$ na fração silte $(2 \leq \mathrm{x} \leq 63 \mu \mathrm{m}), 10,2-73,5 \%$ na fração areia $(63<\mathrm{x} \leq 2000 \mu \mathrm{m})$ e $0,2 \%$ na fração pedregulho (> $2000 \mu \mathrm{m}$ ). Nota-se também que os resíduos apresentam distintos comportamentos granulométricos, dependendo da etapa em que o resíduo é coletado. O resíduo levigação é o mais grosseiro, enquanto que os resíduos diamantado e polimento são os de granulometrias mais finas. Os valores de massa específica real na faixa de $2,52-3,02 \mathrm{~g} / \mathrm{cm}^{3}$, provavelmente refletem as diferentes composições química e mineralógica dos resíduos. 
Tabela 1: Composição química das amostras de resíduos de rochas ornamentais utilizados.

\begin{tabular}{|c|c|c|c|c|}
\hline \multicolumn{5}{|c|}{ Composição Química (\% em peso) } \\
\hline Óxidos & Resíduo Levigação & Resíduo Diamantado & Resíduo Polimento & Resíduo Granalha \\
\hline $\mathrm{SiO}_{2}$ & 67,058 & 92,058 & 67,720 & 53,516 \\
\hline $\mathrm{Al}_{2} \mathrm{O}_{3}$ & 15,072 & 3,258 & 16,207 & 19,405 \\
\hline $\mathrm{K}_{2} \mathrm{O}$ & 6,615 & 0,933 & 6,733 & 8,612 \\
\hline $\mathrm{Fe}_{2} \mathrm{O}_{3}$ & 6,087 & - & 3,447 & 8,059 \\
\hline $\mathrm{CaO}$ & 2,0275 & 1,071 & 3,446 & 6,890 \\
\hline $\mathrm{SO}_{3}$ & 2,021 & 1,725 & 1,620 & 1,574 \\
\hline $\mathrm{TiO}_{2}$ & 0,594 & - & 0,477 & 0,882 \\
\hline $\mathrm{MnO}$ & 0,139 & - & 0,085 & - \\
\hline $\mathrm{Sc}_{2} \mathrm{O}_{3}$ & 0,067 & - & - & - \\
\hline $\mathrm{ZrO}_{2}$ & 0,056 & - & 0,038 & 0,067 \\
\hline $\mathrm{Y}_{2} \mathrm{O}_{3}$ & 0,015 & - & 0,006 & - \\
\hline
\end{tabular}

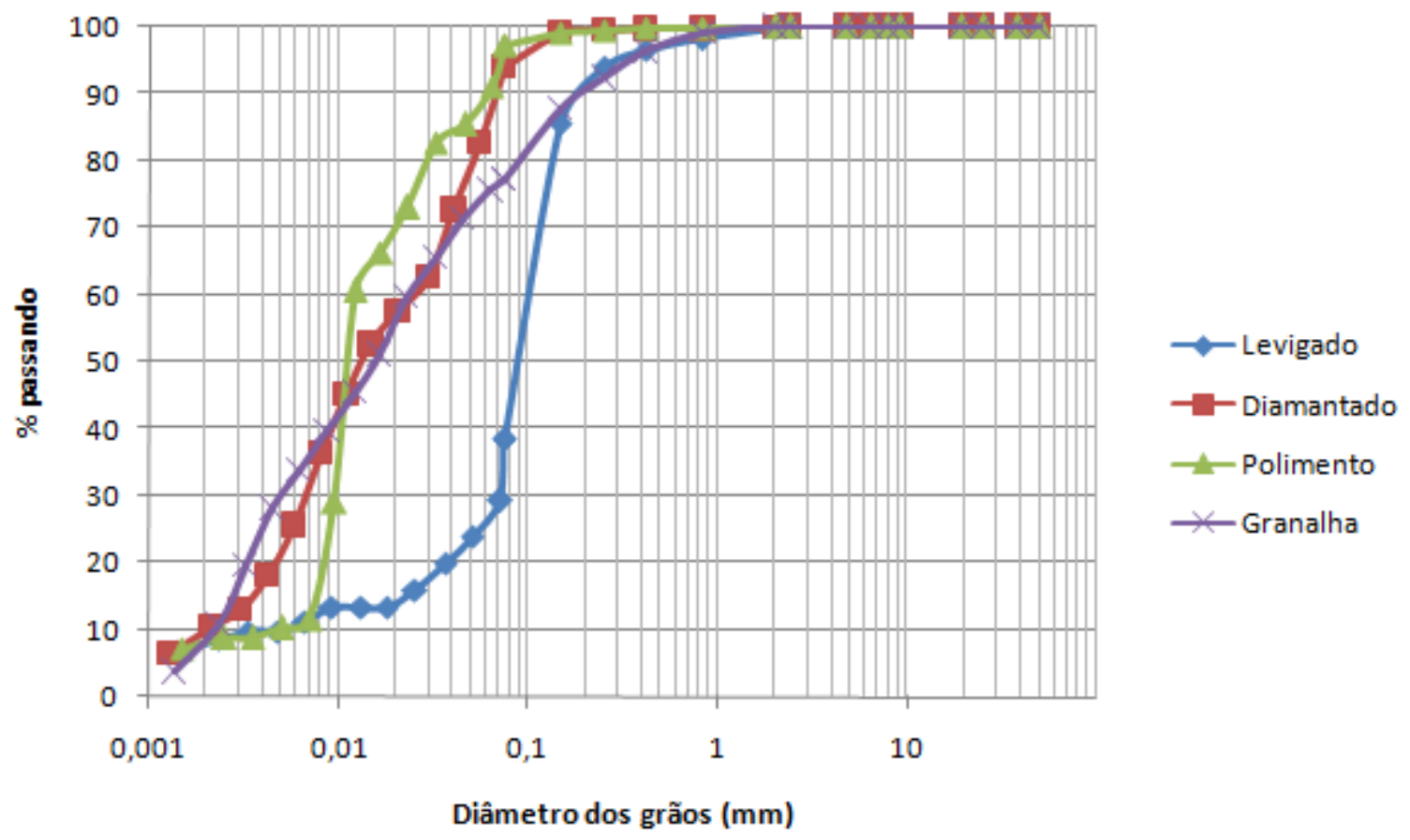

Figura 1: Curvas de distribuição do tamanho das partículas dos resíduos estudados. 
Tabela 2: Dados granulométricos e massa específica real dos resíduos estudados.

\begin{tabular}{c|c|c|c|c}
\hline Resíduo & Diamantado & Granalha & Levigação & Polimento \\
\hline Pedregulho (\%) & 0,2 & - & 0,2 & - \\
\hline Argila (\%) & 9,8 & 8,5 & 7,9 & 8,0 \\
\hline Silte (\%) & 75,6 & 66,3 & 18,4 & 81,8 \\
\hline Areia (\%) & 14,4 & 25,3 & 73,5 & 10,2 \\
\hline $\begin{array}{c}\text { Massa específica } \\
\text { real }\left(\mathrm{g} / \mathrm{cm}^{3}\right)\end{array}$ & 3,02 & 2,82 & 2,70 & 2,52 \\
\hline
\end{tabular}

As Figuras 2-5 apresentam as morfologias obtidas via microscopia eletrônica de varredura dos resíduos de rocha ornamental estudados. De forma geral todas as amostras de resíduos de rochas ornamentais apresentam partículas tipicamente irregulares. Este tipo de formato irregular é típico dos processos de corte e beneficiamento de rochas ornamentais [9]. Pode-se observar ainda nas figuras a presença de partículas de quartzo muito bem delineadas, porém, com formas irregulares e arestas angulares. As micrografias também mostram uma larga distribuição dos tamanhos das partículas para as amostras dos resíduos de rochas ornamentais, o qual corrobora com os dados granulométricos (Tabela 2).

Nas Figuras 6-8 são apresentados os resultados da análise termogravimétrica das amostras de resíduos de rochas ornamentais. Pode-se observar que os resíduos de rochas ornamentais estudados apresentam diferentes comportamentos térmicos. Isto está relacionado às diferentes características destes resíduos como, por exemplo, as diferentes composições química e granulométrica. A curva DTG da Figura 6 indica que o resíduo diamantado apresenta três eventos endotérmicos nas faixas de temperaturas de $\sim 75^{\circ} \mathrm{C}$ (remoção de água fisicamente adsorvida na superfície das partículas), $\sim 600^{\circ} \mathrm{C}$ (referente a transformação $\alpha-\beta$ quartzo) $\mathrm{e} \sim 850^{\circ} \mathrm{C}$ (provavelmente relacionado a desidroxilação do mineral micáceo, geralmente presente em rochas silicáticas[3] ). O resíduo granalha (Fig. 7) apresenta quatro eventos endotérmicos em temperaturas de $\sim 80^{\circ} \mathrm{C}$ (remoção de água fisicamente adsorvida na superfície das partículas), $\sim 50{ }^{\circ} \mathrm{C}$ (referente a transformação $\alpha-$ $\beta$ quartzo), $\sim 775^{\circ} \mathrm{C}$ (provavelmente referente à decomposição de calcita presente neste tipo de resíduo oriunda da cal usada como lubrificante durante o processo de corte) e $\sim 820^{\circ} \mathrm{C}$ (provavelmente relacionado à desidroxilação do mineral micáceo geralmente presente em rochas silicáticas []] ). O resíduo granalha também apresenta maior perda de massa devido a decomposição de calcita e concomitante liberação de $\mathrm{CO}_{2}$. Já o resíduo polimento (Fig. 8) também apresenta três eventos endotérmicos relacionados à remoção de água fisicamente adsorvida na superfície das partículas $\left(\sim 80^{\circ} \mathrm{C}\right)$, transformação $\alpha-\beta$ quartzo $\left(\sim 580^{\circ} \mathrm{C}\right)$ e desidroxilação do mineral micáceo $\left(\sim 800^{\circ} \mathrm{C}\right)$.

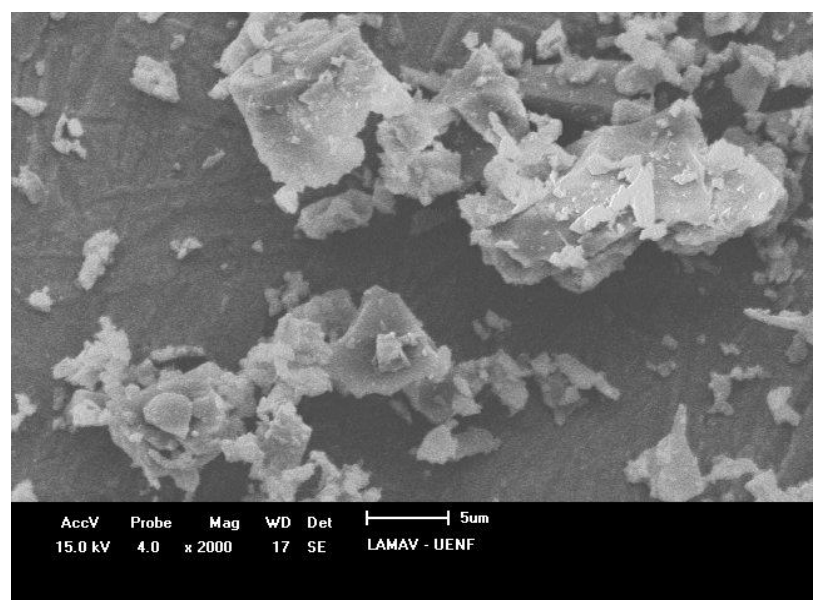

Figura 2: Morfologia das partículas do resíduo diamantado. 


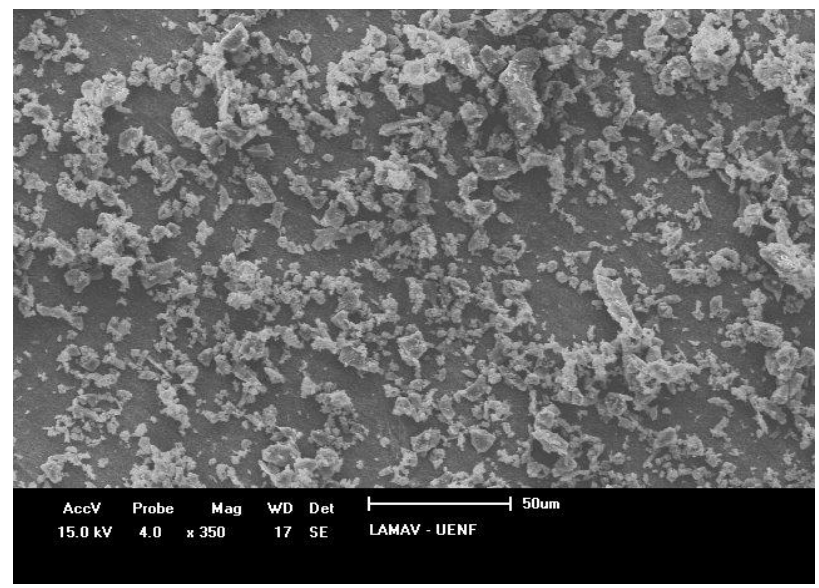

Figura 3: Morfologia das partículas do resíduo granalha.

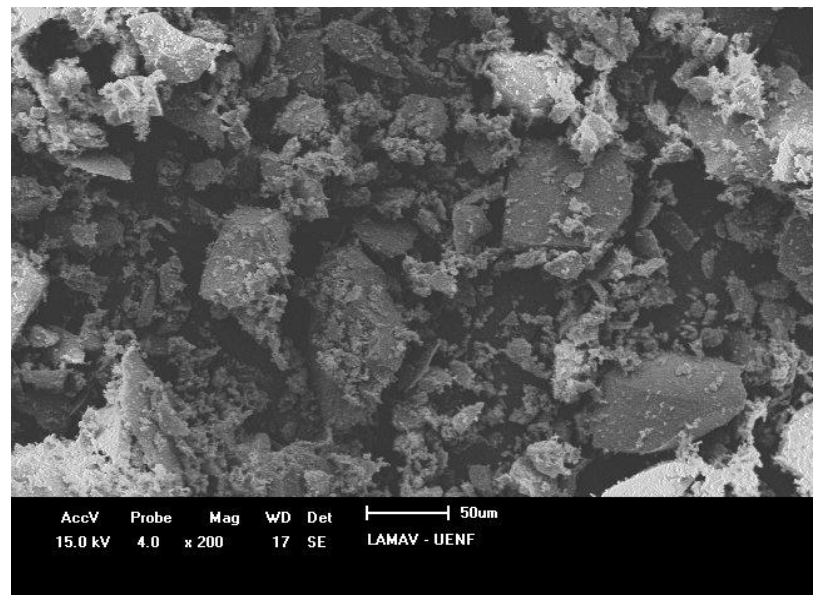

Figura 4: Morfologia das partículas do resíduo levigação.

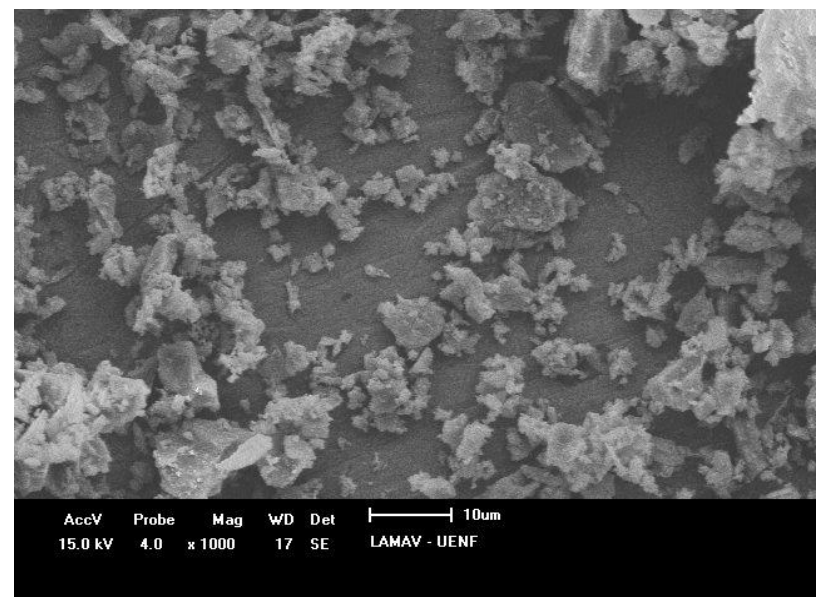

Figura 5: Morfologia das partículas do resíduo polimento. 


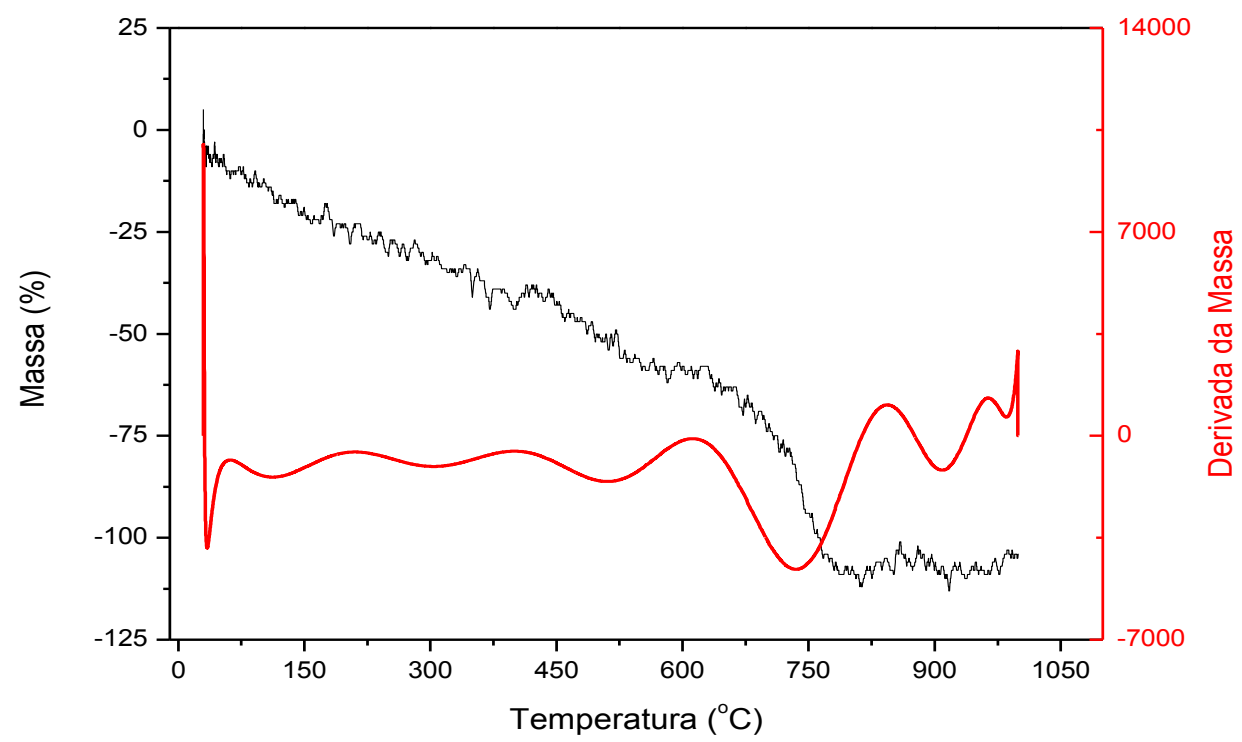

Figura 6: Análise termogravimétrica do resíduo diamantado.

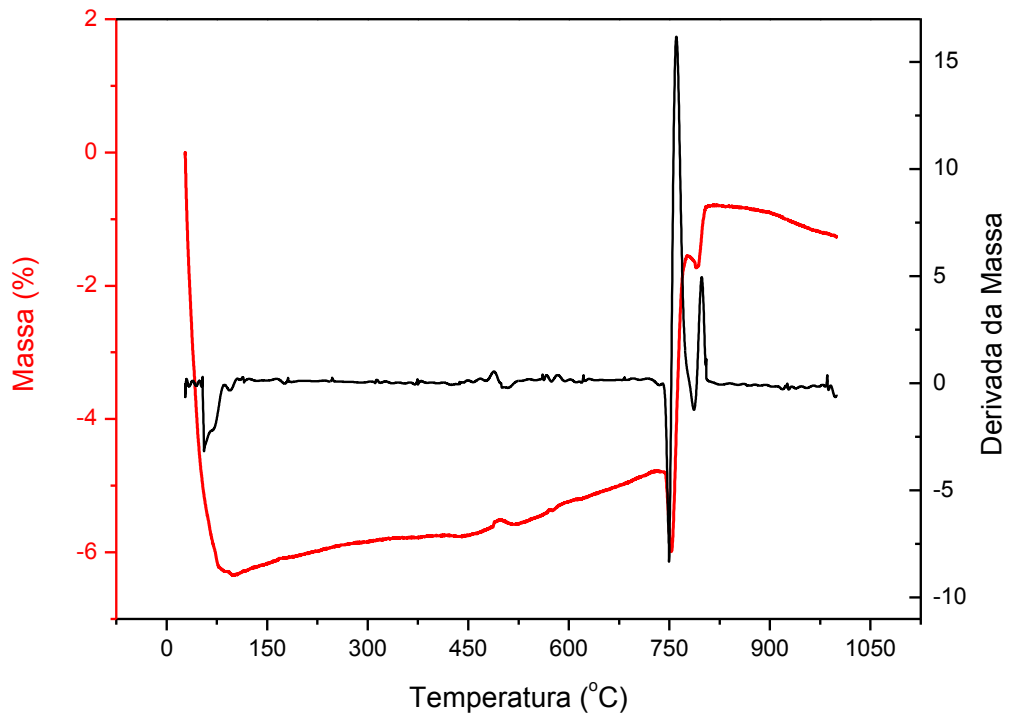

Figura 7: Análise termogravimétrica do resíduo granalha. 


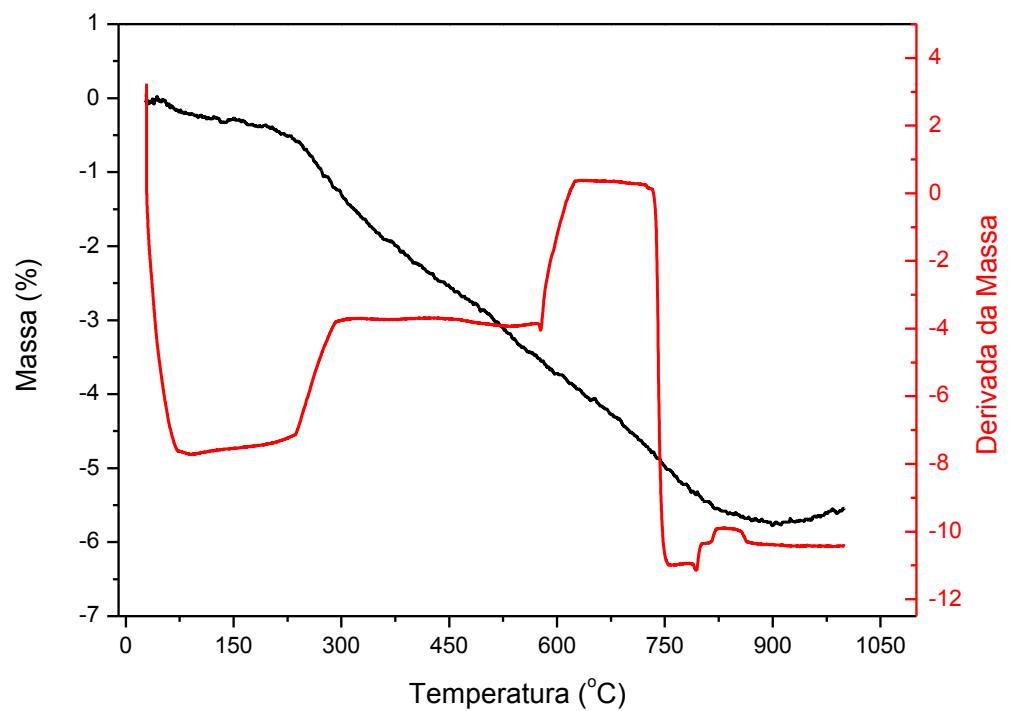

Figura 8: Análise termogravimétrica do resíduo polimento.

\section{CONCLUSÕES}

Os resultados experimentais indicam que os resíduos estudados coletados em diferentes processos de corte e beneficiamento de rochas ornamentais apresentam diferentes características químicas, físicas e térmicas.

Os resultados de composição química mostraram que todos os resíduos têm como principal elemento sílica $\left(\mathrm{SiO}_{2}\right)$, que é característico de rochas ácidas (rochas silicatadas). O resíduo granalha resultante do corte com granalha metálica apresentar o maior teor de óxido de ferro, o qual está relacionado ao uso intensivo de abrasivo para facilitar o processo de corte. A presença de óxido de cálcio no processo de corte com granalha é resultante da adição da cal na lama abrasiva.

As análises granulométricas mostraram que os resíduos apresentam diferentes comportamentos granulométricos. O resíduo gerado do processo de levigação das rochas possui granulometria mais grosseira, enquanto que o resíduo diamantado e resíduo polimento possuem mais finas, inclusive são constituídos de partículas menores que $75 \mu \mathrm{m}$ superiores a $85 \%$.

A análise morfológica de uma forma geral demonstrou para todas as amostras que as partículas dos resíduos de rochas ornamentais são irregulares devido aos processos de corte e beneficiamento ao qual as rochas são submetidas.

Os resultados também indicaram que os resíduos de rochas ornamentais apresentam diferentes comportamentos térmicos, relacionados às diferentes composições.

\section{AGRADECIMENTOS}

Os autores agradecem ao CNPq e FAPERJ pelo apoio financeiro e as empresas Decolores Mármores e Granitos e Pazigran pelo fornecimento dos resíduos de rochas ornamentais.

\section{BIBLIOGRAFIA}

[1] BALDOTTO, Marihus Altoé., ASPIAZU, I., SILVA, A. P., et al.,"H. Potencialidade Agronômica dos resíduos de Rochas Ornamentais"., Revista Capixaba de Ciência Tecnologia, n. 3, p. 1-8, 2007.

[2] PINA, P., SARAIVA, J., BANDEIRA, L. Formação Avançada no Setor de Rochas Ornamentais e Geoprocessamento, Portugal, CERENA/IST, 2009.

[3] DANA, J.D., Mineralogy Handbook, Rio de Janeiro, LTC, 1978.

[4] GUERREIRO, H. J.P., Exploração Subterrânea de Mármores - Aspectos Geotécnicos, Dissertação de M.Sc Geotecnia., Universidade Técnica de Lisboa/Instituto Superior Técnico Portugal, Lisboa, 2000.

[5] ABIROCHAS, informe 11/2012, http://www.abirochas.com.br/. Acessado em Abril de 2012. 
[6] ABIROCHAS, http://www.abirochas.com.br/. Acessado em Abril de 2012.

[7] ABIROCHAS, http://www.abirochas.com.br/rochas_ornamentais_04.php. Acessado em Outubro de 2010.

[8] ABIROCHAS - Associação Brasileira da indústria de Rochas Ornamentais. Situação Atual e Perspectivas Brasileiras no Setor de Rochas Ornamentais, 2010.

[9] MANHÃES, J.P.V.T., HOLANDA, J.N.F., "Caracterização e Classificação de resíduo Sólido Pó de Rocha Granítica Gerado na Indústria de Rochas Ornamentais”, Química Nova, v. 31, n.6, pp. 1301-1304, 2008.

[10] TORRES, P., FERNANDES, H.R., OLHEIRO, S., et al., "Incorporation of Wastes Granite Rock Cutting and Polishing Industries to Produce Roof Tiles", Journal of European Ceramic Society, v. 29, n.1, pp. 23-30, 2009.

[11] MONTEIRO, M.A., JORDAN, M.M., ALMENDRO-CANDEL, M.B.,et al., "The Use of a Calcium Carbonate Residue from the Stone Industry and Manufacturing of Ceramic Tile Bodies", Applied Clay Science, v. 43, n. 2, pp. 186- 189, 2009.

[12] SOUZA, A.J., PINHEIRO, B.C.A., HOLANDA, J.N.F., "Recycling of Gneiss Rock Waste in the Manufacture of Vitrified Floor Tile", Journal of Environmental Management, v. 91, n.3, pp. 685-689, 2010.

[13] MOURA, W.A., LEITE, M.B., "Estudo da Viabilidade da Produção de Blocos com Utilização de Resíduo de Serragem de Rochas Ornamentais para Alvenaria de Vedação”, Revista da Escola de Minas, v. 64, n. 2, pp. 147-154, 2011.

[14] ASSOCIAÇÃO BRASILEIRA DE NORMAS TÉCNICAS - ABNT. 1987. Amostragem de Resíduos NBR 10007. Rio de Janeiro.

[15] ASSOCIAÇÃO BRASILEIRA DE NORMAS TÉCNICAS - ABNT. 1984. Análise Granulométrica NBR 7181. Rio de Janeiro.

[16] ASSOCIAÇÃO BRASILEIRA DE NORMAS TÉCNICAS - ABNT. 2003. Agregado Miúdo - Determinação da Massa Específica e Massa Específica Aparente - NBR NM 52. Rio de Janeiro.

[17] CARTY, W.M., SENGUPTA, U., "Porcelain - Raw Materials, Processing, Phase Evolution, and Mechanical Properties", Journal of The American Ceramic Society, v. 81, n. 1, pp. 3-20, 1998.

[18] BARBA, A., FELÍU, C., GARCIA, J.,et al., Matérias Primas para la Fabricación de Soportes de Baldosas Cerámicas, 2 ed., Castellón, Espanha, ITC, 2002.

[19] SOUZA, A.J. Estudo da substituição de fundente natural por resíduo de rocha ornamental em massa cerâmica para piso vitrificado, Dissertação de Mestrado, UENF-PPGECM, Campos dos Goytacazes-RJ, 2008.

[20] MOURA, W.A., GONÇALVES, J.P., LEITE, R.S., "Utilização de Resíduo de Corte de Mármore e Granito em Argamassa de Revestimento e Confecção de lajotas para Piso”, Sitientibus, n. 26, pp. 49-61, 2002.

[21] DING, Y., JALALI, S., NIEDEREGGER, C. "Recycling of Metamorphic Rock Waste in Ecological Cement”, Construction Materials, v. 163, n. 3, pp. 143-148, 2010. 